\title{
Vector subgroupoids. The computation of all vector subgroupoids of a modular vector groupoid
}

\section{GHEORGHe IVAN and VAsile PopuţA}

\section{ABSTRACT.}

A vector groupoid is an algebraic structure which combines the concepts of groupoid and vector space such that these are compatible. In this paper we study the vector subgroupoids. Also, the vector subgroupoids of a modular vector groupoid are computed.

DEPARTMENT OF MATHEMATICS

WEST UNIVERSITY OF TIMIŞOARA

V. PÂRVAN 4, 300223 TIMIŞOARA, ROMANIA

E-mail address: ivan@math. uvt.ro

E-mail address: vpoputa@yahoo.com 